\title{
VENTRICULAR WEIGHT IN CARDIAC HYPERTROPHY
}

BY

\author{
R. M. FULTON, E. C. HUTCHINSON, AND A. MORGAN JONES \\ From Crumpsall Hospital, Manchester and the Department of Cardiology, University of Manchester
}

Received January 30, 1952

\begin{abstract}
Ventricular hypertrophy is usually estimated at necropsy by weighing the heart as a whole and by measuring the thickness of the ventricular walls. The weight of the heart as a whole is open to the objection that a large and varying proportion of the weight is accounted for by structures other than the myocardium, and the thickness of the ventricular wall is not a satisfactory criterion because it may be greatly modified by dilatation of the cavity. The traditional methods are particularly unsatisfactory when used to assess slight or moderate hypertrophy of the right ventricle.

In an attempt to overcome these difficulties, Müller (1883), Lewis (1914), and Herrmann and Wilson (1922) stripped off non-myocardial tissue and subsequently divided the myocardium into right and left parts. Their techniques are time-consuming, however, and the methods of dividing the septum are open to question so that they have not come into general use. The present investigation is an attempt to evolve a simpler technique for measuring hypertrophy of each ventricle separately, with the special object of assessing lesser degrees of right ventricular hypertrophy.
\end{abstract}

\section{METHODS}

Technique and Division of Hearts. The method described here is a modification of that employed by Lewis (1914). From the results obtained with this method, the simpler technique to be described later was developed. The heart, aorta, and lungs were removed intact from the thorax: the lungs were then separated from the heart, leaving as much as possible of the pulmonary vessels attached to the heart. After inspection for congenital and other lesions of the great vessels, the aorta and pulmonary artery were divided about $1 \mathrm{~cm}$. above the semilunar valves, which were examined. The atria were then opened posteriorly, and the blood clot washed out from the atrial and ventricular cavities. After examination of the mitral and tricuspid valves and the interior of the atria, the heart was packed with cotton wool to maintain its shape, and immersed in 10 per cent formol saline for 72-96 hours. At the end of this time, the coronary arteries were dissected free and opened up. The heart was stripped of vessels and external fat by sharp dissection, leaving the myocardium exposed, and the cotton wool packing was removed. The atria were separated from the ventricles at the atrio-ventricular ring, leaving the isolated ventricular myocardium.

The tendon of the infundibulum was next divided, allowing the pulmonary conus to fall away from the septum, and exposing the anterior junction of the free wall of the right ventricle and the septum The free wall of the right ventricle was separated from the septum with scissors, beginning at the anterior base of the septum and cutting down to the apex, and up the posterior margin of the septum. The cut was made so that the cut surface was in the same plane as the right ventricular surface of the septum. The junction of the comparatively thin right ventricular wall with the septum was easily defined and usually formed an acute angle. Separation was easy, and there was little opportunity for personal error. Trabeculæ on the right ventricular aspect of the septum were cut off at their base: this involved additional trimming when the trabeculæ were hypertrophied, as they were in hearts with marked right ventricular hypertrophy. In a similar way, the free wall of the left ventricle was separated from the septum, but this was more difficult, for the left ventricle and septum form an almost circular band of muscle of roughly the same thickness and the junction of the two is ill-defined. The final cut surface was in the same plane as the flat central portion of the left ventricular aspect of the septum, but additional trimming was always required, and there was room for appreciable personal error. Finally, the valve cusps and the remaining parts of the aorta and pulmonary artery were removed and the three pieces of myocardium (free wall of right ventricle, free wall of left ventricle, and the septum) were weighed on a balance, accurate to $0.5 \mathrm{~g}$. 
The possibility of a change in weight due to fixation was considered. Müller (1883) obviated this possibility by dividing the heart in a fresh state, but this makes the dissection much more difficult. Lewis (1914) took great care to restore the original weight of the heart after fixation by successive immersions in formalin, spirit, and water. Herrmann and Wilson (1922) found that prolonged immersion in formalin could cause a loss of weight of over 10 per cent. In our series, however, fixation was not continued for more than four days and in that time the weight of the cardiac muscle did not vary by more than 2 per cent. Consequently, we have felt justified in ignoring the effects of fixation upon the recorded weights.

\section{Classification of Material}

We examined 202 hearts and classified them into 4 groups. The basis of the classification was a clinical one, but the diagnosis of valvular lesions and of myocardial infarction was subject to necropsy confirmation.

Group 1. Normal (43 hearts). There was no evidence of heart disease either clinically or at necropsy. Hearts from patients over 65 years of age were not included in this group.

Group 2. Isolated Right Ventricular Hypertrophy (46 hearts). There was a clinical cause of hypertrophy of the right ventricle alone. The cases comprised chronic cor pulmonale * (37), isolated mitral stenosis (7), and mitral stenosis with a tricuspid lesion (2).

Group 3. Primary Left Ventricular Hypertrophy (46 hearts). A cause of primary left ventricular hypertrophy was present clinically, but there was no known cause of independent right ventricular hypertrophy. The cases comprised hypertension (38), isolated aortic lesions (6), and pure mitral incompetence (2).

Group 4. Miscellaneous (67). This group consisted of apparently normal hearts from patients over 65 years of age (33), cases where there were lesions liable to cause independent hypertrophy of both ventricles (10), myocardial infarction (6), and unclassified cases (18). It soon became clear that the cases in this group were unsuitable for detailed study, and as the investigation progressed, only hearts that could be included in the first three groups were dissected.

\section{RESULTS}

Group 1. Normal Hearts. Fig. 1 shows the distribution curves of the total ventricular weight and the weights of the three sub-divisions of the myocardium in the normal group. Table I shows the maximum, minimum, and mean weights with suggested upper limits of normality for each part.

Group 2. Isolated Right Ventricular Hypertrophy. This group was divided into patients who died with congestive heart failure (35) and those who died from some other cause (11). Table II shows the maximum, minimum, and mean weights of the various parts of the heart in this group, and indicates the frequency with which each part was above the normal maximum weight.

TABLE I

The Range of Weight in Normal Hearts

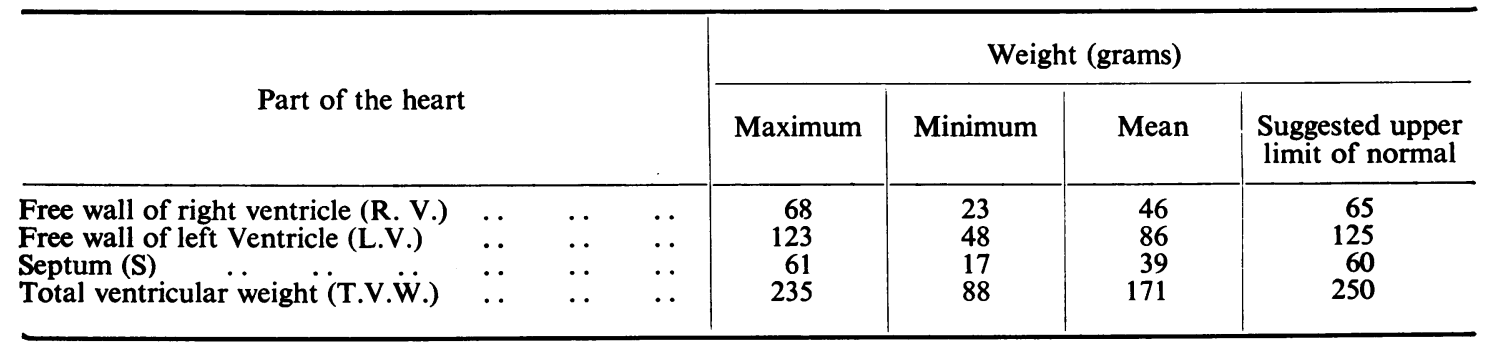

* The striking preponderance of cases of chronic cor pulmonale was due to the nature of the hospital material, the fact that the investigation took place during the winter months, and that a concurrent investigation concerning chronic cor pulmonale led to a very high necropsy rate in fatal cases. 


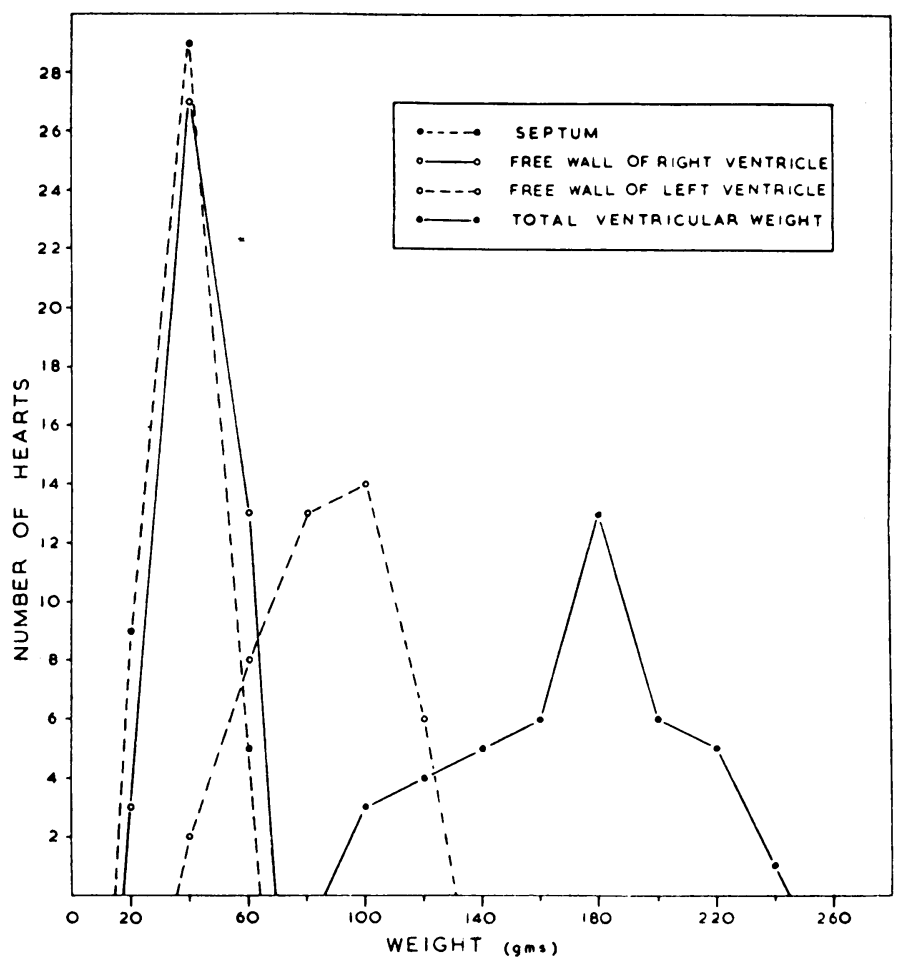

FIG. 1.-Normal hearts: Distribution curves of the total ventricular weight and the weights of the three sub-divisions of the myocardium.

TABLE II

The Range of Weight in Right and Left Ventricular Hypertrophy

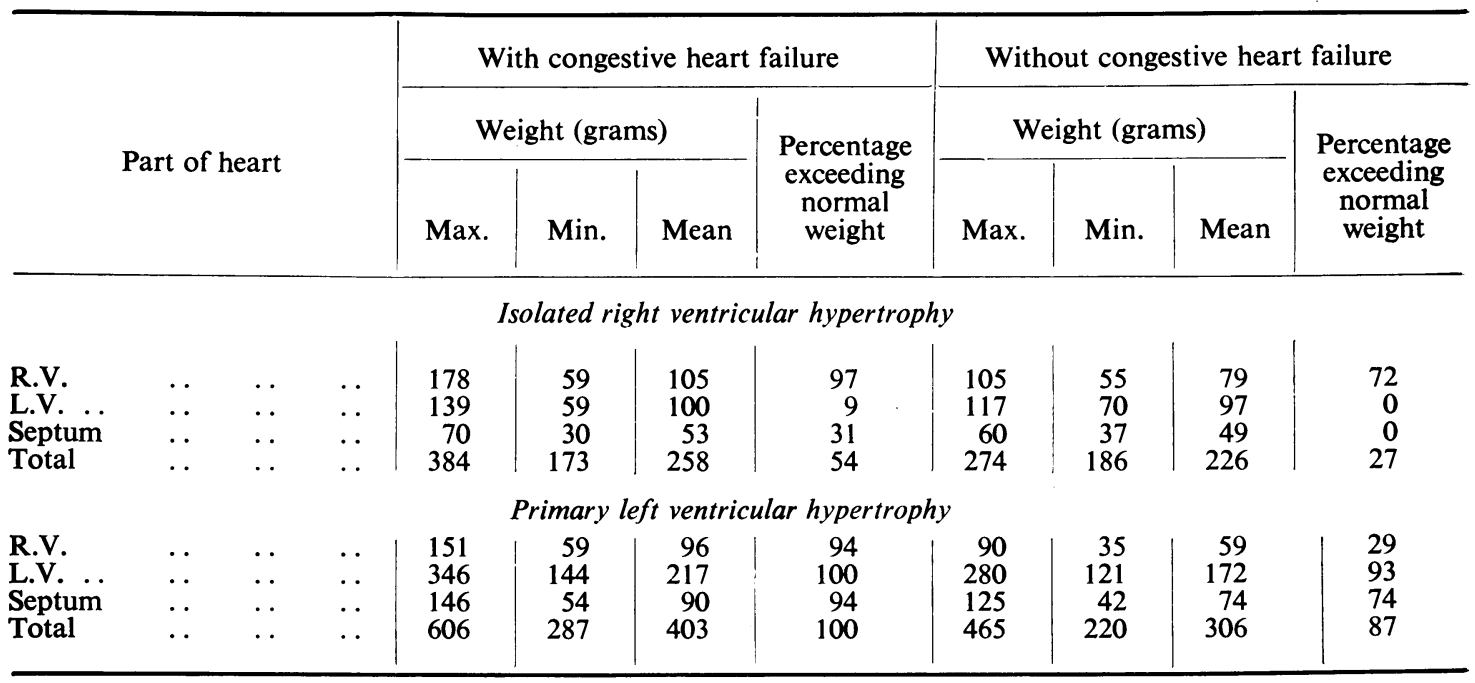


Group 3. Primary Left Ventricular Hypertrophy. This group was similarly divided into patients who died with (15) and without (31) congestive heart failure. Table II shows the maximum, minimum, and mean weights of the various parts of the heart, indicating the frequency with which each part was overweight.

\section{Discussion}

Normal Hearts. The average normal weights in the present series correspond fairly closely with those of other workers (Table III). Both Lewis (1914), and Herrmann and Wilson (1922) found that the normal range for the ratio of left to right ventricular weight was from $1.5: 1$ to $2 \cdot 2: 1$, a range which includes all but three of our cases. Neither, however, gave a range of normal for the actual weights of the separate parts of the myocardium. As the ratio may in itself be misleading (as, for example, when there is hypertrophy of both ventricles giving a normal ratio), it is essential to consider the actual weights in coming to a decision about the presence or degree of hypertrophy.

Isolated Right Ventricular Hypertrophy. Hypertrophy was found to be practically confined to the free wall of the right ventricle in this group (Fig. 2, Table II). Even in cases with failure, the total ventricular weight was only above the normal range in about half the cases, and the left ventricle and septum were overweight in only 9 and 31 per cent of cases respectively, and then only to a slight extent.

Primary Left Ventricular Hypertrophy. Increase in the weight of the left ventricle was associated with an increase in the weight of the septum and in the total ventricular weight (Fig. 2B, Table II). In cases with failure, the right ventricle was overweight in almost every case, and even in cases without

A HEARTS WITH ISOLATED RIGHT VENTRICULAR HYPERTROPHY
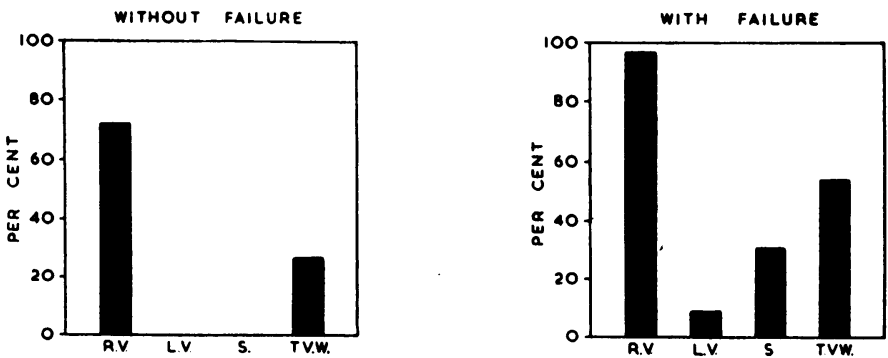

B

HEARTS WITH PRIMARY LEFT VENTRICULAR HYPERTROPHY
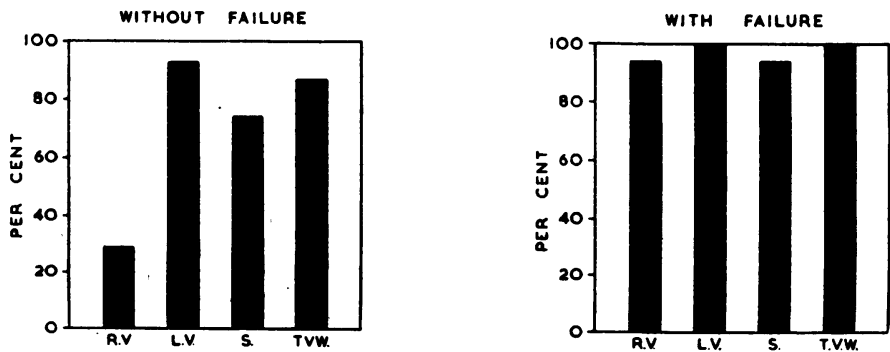

Fig. 2.-The heights of the black columns indicate the percentage of cases in which the weight of the corresponding part of the myocardium exceeded the upper limit of normal. (A) In hearts with isolated right ventricular hypertrophy. (B) In hearts with pulmonary left ventricular hypertrophy. R.V.-right ventricle. L.V.-left ventricle. S-septum. T.V.W. - total ventricular weight. 
TABLE III

Average Weights in Present series Compared with those of Previous Workers

\begin{tabular}{|c|c|c|c|c|c|c|c|}
\hline \multirow{2}{*}{ Author } & \multirow{2}{*}{$\begin{array}{l}\text { No. of } \\
\text { cases }\end{array}$} & \multirow{2}{*}{$\begin{array}{l}\text { Average } \\
\text { age } \\
\text { (years) }\end{array}$} & \multicolumn{4}{|c|}{ Average weight (grams) } & \multirow{2}{*}{$\begin{array}{c}\text { Average } \\
\text { ratio } \\
\text { L.V.: } \mathbf{R . V} \text {. }\end{array}$} \\
\hline & & & R.V. & L.V. & Septum & Total & \\
\hline $\begin{array}{l}\text { Lewis (1914) } \\
\text { Herrmann and Wilson }(1922) * \\
\text { Present Series }\end{array}$ & $\begin{array}{l}16 \\
23 \\
43\end{array}$ & $\begin{array}{l}33 \\
41 \\
49\end{array}$ & $\begin{array}{l}49 \\
45 \\
46\end{array}$ & $\begin{array}{l}87 \\
82 \\
86\end{array}$ & $\begin{array}{l}23 \\
25 \\
39\end{array}$ & $\begin{array}{l}159 \\
152 \\
171\end{array}$ & $\begin{array}{l}1 \cdot 74: 1 \\
1 \cdot 81: 1 \\
1 \cdot 9: 1\end{array}$ \\
\hline
\end{tabular}

* Allowance has been made for an average loss of $18 \mathrm{~g}$. in total ventricular weight due to prolonged storage in formalin.

failure, the right ventricle was above the upper limit of normal in about one-third of the cases although there was no independent cause of right ventricular hypertrophy. This bears out the finding of Thompson and White (1936) that the commonest cause of right ventricular hypertrophy is left ventricular failure, and our figures illustrate the development of right ventricular hypertrophy in such cases before right heart failure occurs.

Total Ventricular Weight as a guide to Hypertrophy. From the results of this investigation, a total ventricular weight of over $250 \mathrm{~g}$. may be taken as evidence of cardiac hypertrophy. If left ventricular hypertrophy is suspected, this criterion alone is sufficiently accurate, for practically all cases of suspected left ventricular hypertrophy had a weight greater than $250 \mathrm{~g}$., and the amount by which this weight was exceeded paralleled the degree of left ventricular hypertrophy present. If, however, right ventricular hypertrophy alone is suspected, the total ventricular weight is of little value. Even when isolated right ventricular hypertrophy led to congestive heart failure, the total ventricular weight was within normal limits (less than $250 \mathrm{~g}$.) in half the cases. The total heart weight as usually estimated at necropsy is, of course, even more misleading, as it may include up to $100 \mathrm{~g}$. of non-myocardial tissue. We regard separate weighing as the only accurate way of estimating isolated right ventricular hypertrophy.

The Septum in Cardiac Hypertrophy. The septal weight exceeded the upper limit of normality ( $60 \mathrm{~g}$.) in only 31 per cent of cases of isolated right ventricular hypertrophy with failure, and in cases without failure, the septal weight was invariably within normal limits (Fig. 2, Table II). The increase in weight when present was only slight, and in no case did the septum weigh more than 70 g. (Fig. 3). In hearts with left ventricular hypertrophy, on the other hand, the septum was overweight in 80 per cent of all cases (Fig. 2, Table II) and the increase in weight was often considerable (Fig. 3). Thus in our cases of acquired heart disease, striking septal hypertrophy occurred only in association with left ventricular hypertrophy. We do not suggest that this is universally true, for it is a matter of common observation that when very great right ventricular hypertrophy occurs in congenital heart disease, the septum may also be greatly hypertrophied. From the data in our cases there is no reason to suppose that the septum does not increase in proportion to the increase in the weight of the free wall of either ventricle. The apparent discrepancy illustrated in Fig. 3 can be explained by the fact that in our cases the increase of weight of the right free wall is usually much smaller than the increase of weight of the left free wall.

In any method of dividing the heart into right and left sides, the allocation of the septal weight has proved difficult. Müller divided the septal weight between right and left sides in proportion to the weight of the free walls. Lewis included a slice of septum with both ventricles, giving two almost completely closed cavities and a small strip of septum. (This accounts for his average septal weight being much less than ours). In comparing the weights of the two ventricles, he ignored the strip of septum. Herrmann and Wilson, in their method B, cut serial horizontal sections of the ventricles, and divided each slice into a right and left half, cutting along a white line in the septum, 


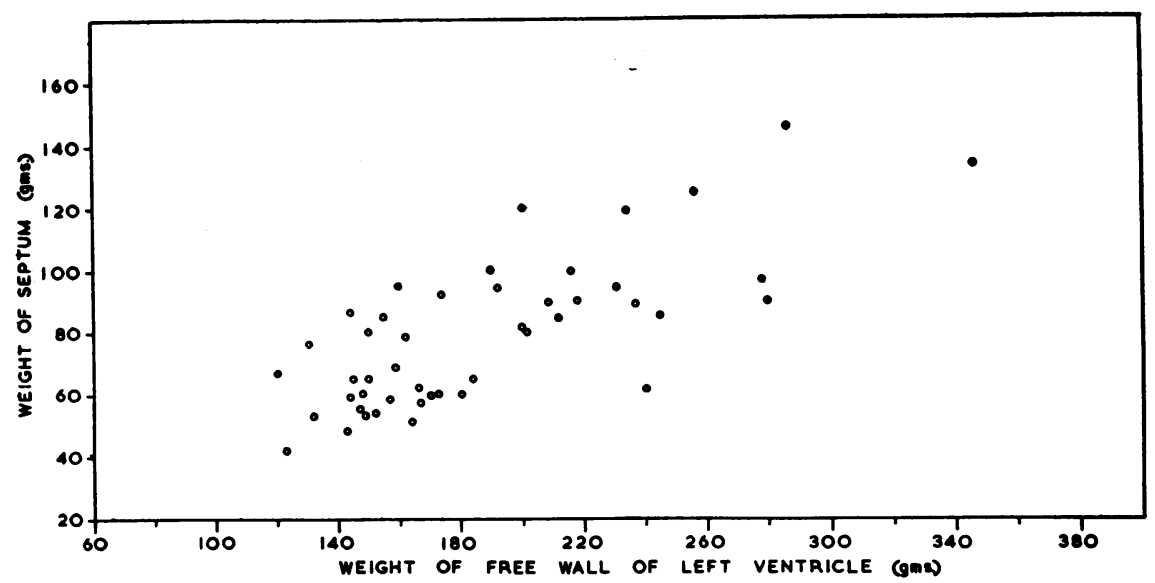

A. HEARTS WITH PRIMARY LEFT VENTRICULAR HYPERTROPHY

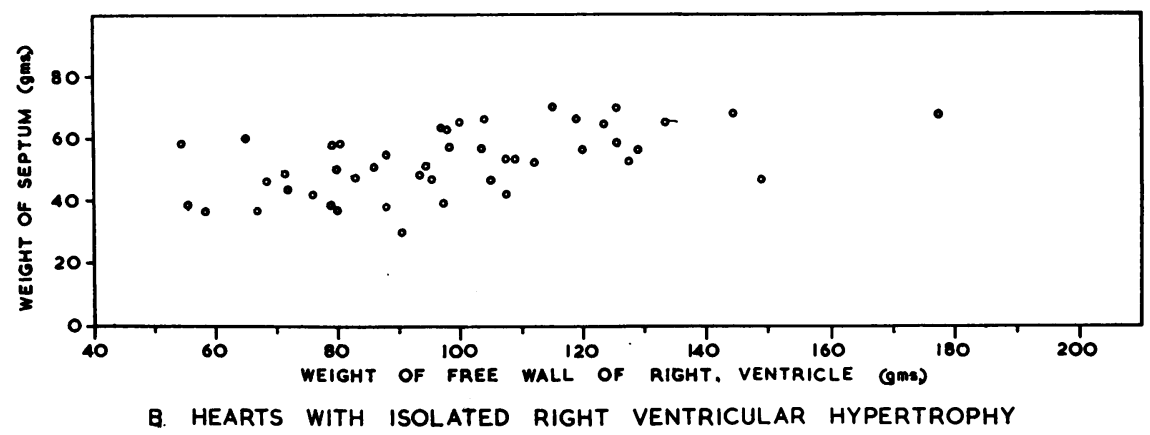

FIG. 3.-Septal weights in (A) Primary left ventricular hypertrophy, and (B) Isolated right ventricular hypertrophy.

which, in their opinion, divided the septum into two halves belonging functionally to the right and left ventricle. This white line, however, if followed backwards and forwards can be traced right round the left ventricle some distance inside the epicardial surface. Herrmann and Wilson's assumption may not be true.

In view of our finding, that in acquired heart disease the septum was never greatly increased in weight except in the presence of left ventricular hypertrophy, it seemed reasonable to consider the free wall of the left ventricle and the septum together as one part of the heart. This has the great advantage of technical simplicity in division, as separation of the free wall of the right ventricle from the rest of the heart is easy and accurate, whereas separation of the free wall of the left ventricle from the septum is difficult and open to error.

The upper limit of normal weight of the free wall of the left ventricle and septum together we regard as $190 \mathrm{~g}$., and the normal range for the ratio of left ventricular plus septal weight to the weight of the free wall of the right ventricle $(L+S / R)$ was found to be from $2 \cdot 3: 1$ to $3 \cdot 3: 1$.

Consideration of the heart weights in the present series in this simplified way shows satisfactory agreement with the results of the more detailed dissection. It is suggested, therefore, that after fixation, the free wall of the right ventricle should be separated from the rest of the heart as already described, and that the two pieces-first the free wall of right ventricle and secondly the free wall of left ventricle and septum together-should then be weighted. Using this method our criteria for normality and for right and left ventricular hypertrophy are as follows. 

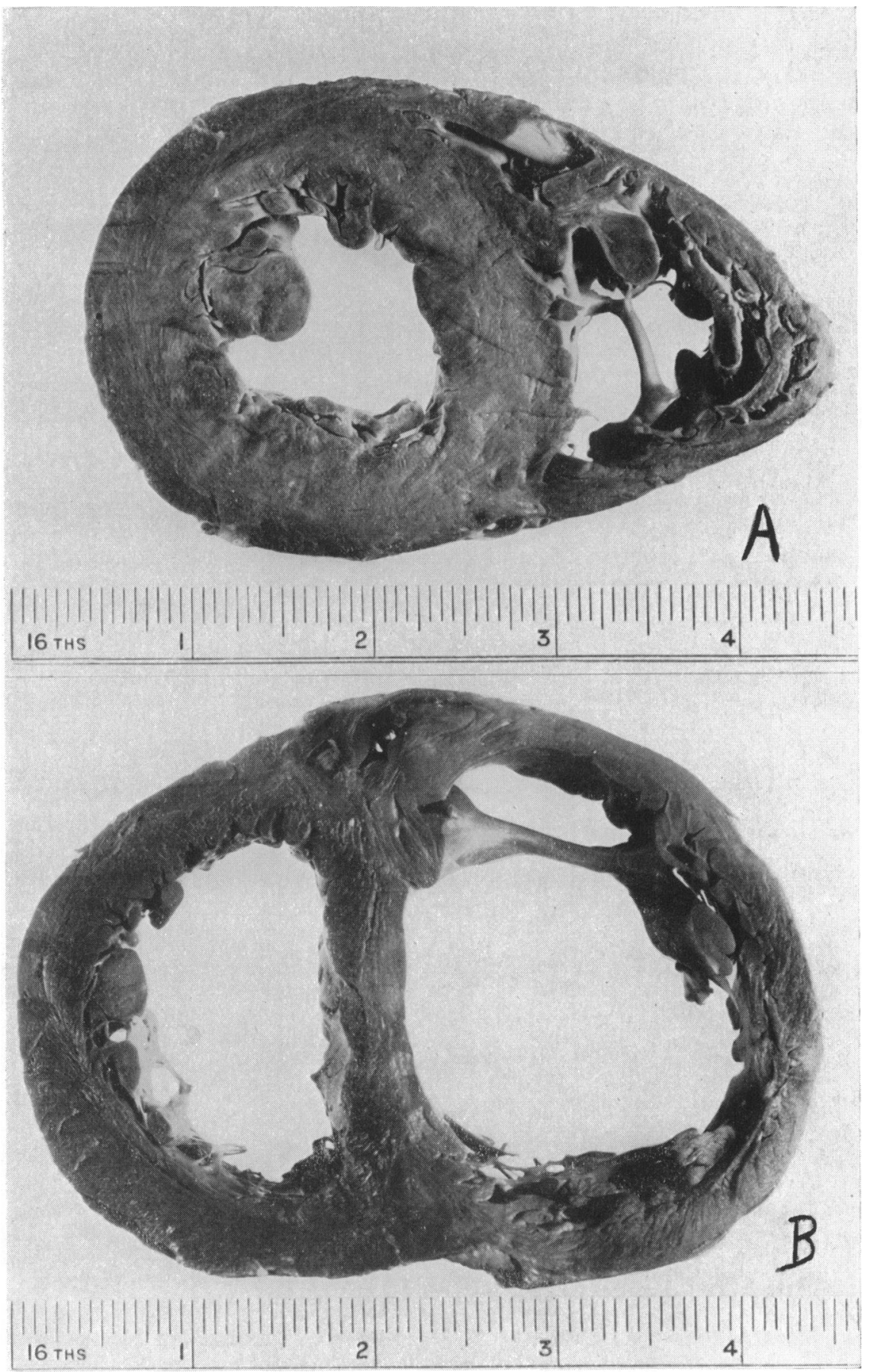

FIG. 4.-Transverse sections through two hearts. (A) with primary left ventricular hypertrophy, and (B) with isolated right ventricular hypertrophy. 
Criteria for Normality. A heart may be classed as normal only if

(a) the total ventricular weight is less than $250 \mathrm{~g}$.,

(b) the free wall of the right ventricle weighs less than $65 \mathrm{~g}$.,

(c) the left ventricle and septum together weigh less than $190 \mathrm{~g}$., and

(d) the ratio $\mathrm{L}+\mathrm{S} / \mathrm{R}$ lies between $2 \cdot 3: 1$ and $3 \cdot 3: 1$.

Criteria for Right Ventricular Hypertrophy. Right ventricular hypertrophy is considered to be present when the free wall of the right ventricle weighs $80 \mathrm{~g}$. or more. In isolated right ventricular hypertrophy the ratio $\mathrm{L}+\mathrm{S} / \mathrm{R}$ is always less than $2: 1$. If left ventricular hypertrophy is also present, the ratio may be within normal limits or even raised.

Criteria for Left Ventricular Hypertrophy. Left ventricular hypertrophy is considered to be present when the weight of the left ventricle plus septum is $225 \mathrm{~g}$. or more. The ratio L+S/R may be modified by secondary or independent right ventricular hypertrophy and the ratio alone is therefore not an indication either of the presence or degree of left ventricular hypertrophy.

\section{SUMMARY}

In an attempt to find a satisfactory way of estimating ventricular hypertrophy, 202 hearts were examined. The total ventricular weight and the weights of the free walls of right and left ventricles and of the septum are discussed in relation to normal hearts and hearts with isolated right ventricular hypertrophy and primary left ventricular hypertrophy.

In hearts with left ventricular hypertrophy, the total ventricular weight is always above normal, and is a measure of the degree of left ventricular hypertrophy present. In hearts with isolated right ventricular hypertrophy, the total ventricular weight is within normal limits in 50 per cent of cases, even when congestive failure has been present.

Septal hypertrophy is much commoner and more pronounced in left ventricular hypertrophy than in right.

A simple separation of the heart into two parts, $(a)$ the free wall of right ventricle and $(b)$ the free wall of left ventricle and septum together, appears to give as accurate an assessment of the site and degree of ventricular hypertrophy as is possible by more elaborate methods.

Criteria for the recognition of normality and of right and left ventricular hypertrophy are suggested.

We wish to acknowledge our indebtedness to Dr. J. V. Davson, Pathologist to the North Manchester Group of Hospitals, and to his Staff at Crumpsall Hospital, for most helpful co-operation which made this investigation possible.

\section{REFERENCES}

Herrmann, G. R., and Wilson, F. N. (1922). Heart, 9, 91.

Lewis, T. (1914). Heart, 5, 367.

Müller, W. (1833). Die Massenverhaltnisse der menshlichen Herzens. Leopold Voss, Hamburg and Leipzig.

Thompson, W. P., and White, P. D. (1936). Amer. Heart J., 12, 641. 